\title{
The HST/WFC3 Quicklook Project: A User Interface to Hubble Space Telescope Wide Field Camera 3 Data
}

\section{Matthew Bourque $^{1}$, Varun Bajaj ${ }^{1}$, Ariel Bowers ${ }^{1}$, Michael Dulude ${ }^{1}$, Meredith Durbin ${ }^{2}$, Catherine Gosmeyer ${ }^{1}$, Heather Gunning ${ }^{1}$, Harish Khandrika ${ }^{1}$, Catherine Martlin ${ }^{1}$, Ben Sunnquist ${ }^{1}$ and Alex Viana ${ }^{3}$}

\author{
${ }^{1}$ Space Telescope Science Institute, \\ 3700 San Martin Drive, Baltimore, Maryland, USA 21218 \\ email: bourque@stsci.edu \\ ${ }^{2}$ Dept. of Astronomy, The University of Washington, \\ Box 351580, U.W. Seattle, Washington, USA 98195 \\ ${ }^{3}$ Terbium Labs, \\ Baltimore, Maryland, USA
}

\begin{abstract}
The Hubble Space Telescope's Wide Field Camera 3 (WFC3) instrument, comprised of two detectors, UVIS (Ultraviolet-Visible) and IR (Infrared), has been acquiring 50-100 images daily since its installation in 2009. The WFC3 Quicklook project provides a means for instrument analysts to store, calibrate, monitor, and interact with these data through the various Quicklook systems: (1) a 175 TB filesystem, which stores the entire WFC3 archive on disk, (2) a MySQL database, which stores image header data, (3) a Python-based automation platform, which currently executes 22 unique calibration/monitoring scripts, (4) a Python-based code library, which provides system functionality such as logging, downloading tools, database connection objects, and filesystem management, and (5) a Python/Flask-based web interface to the Quicklook system. The Quicklook project has enabled large-scale WFC3 analyses and calibrations, such as the monitoring of the health and stability of the WFC3 instrument, the measurement of $\sim 20$ million WFC3/UVIS Point Spread Functions (PSFs), the creation of WFC3/IR persistence calibration products, and many others.
\end{abstract}

Keywords. Hubble Space Telescope, HST, Wide Field Camera 3, WFC3, Automation, Database, Filesystem, Website, Python, Quicklook

\section{Introduction}

Since its installation during Servicing Mission 4 (SM4) in 2009, the Hubble Space Telescope (HST) Wide Field Camera 3 (WFC3) instrument has been acquiring dozens of images daily. The WFC3 'Quicklook' project was adapted shortly after on-orbit observations began as a means to visually inspect new WFC3 data quickly and easily via JPEG images. Since then, the Quicklook project has steadily grown into a robust system that, in addition to the visual inspection of images, allows for the monitoring of the WFC3 instrument's health and stability, the easy accessibility to all on-orbit WFC3 data, and the automated execution of WFC3 calibration and monitoring scripts. This is accomplished by the various Quicklook 'subsystems' (further described in Bourque et al. 2016): (1) a filesystem that stores all on-orbit WFC3 data, (2) a database that stores FITS header metadata for each image, (3) an automation platform that executes calibration and monitoring Python scripts on a daily or weekly basis, (4) a Python code base 


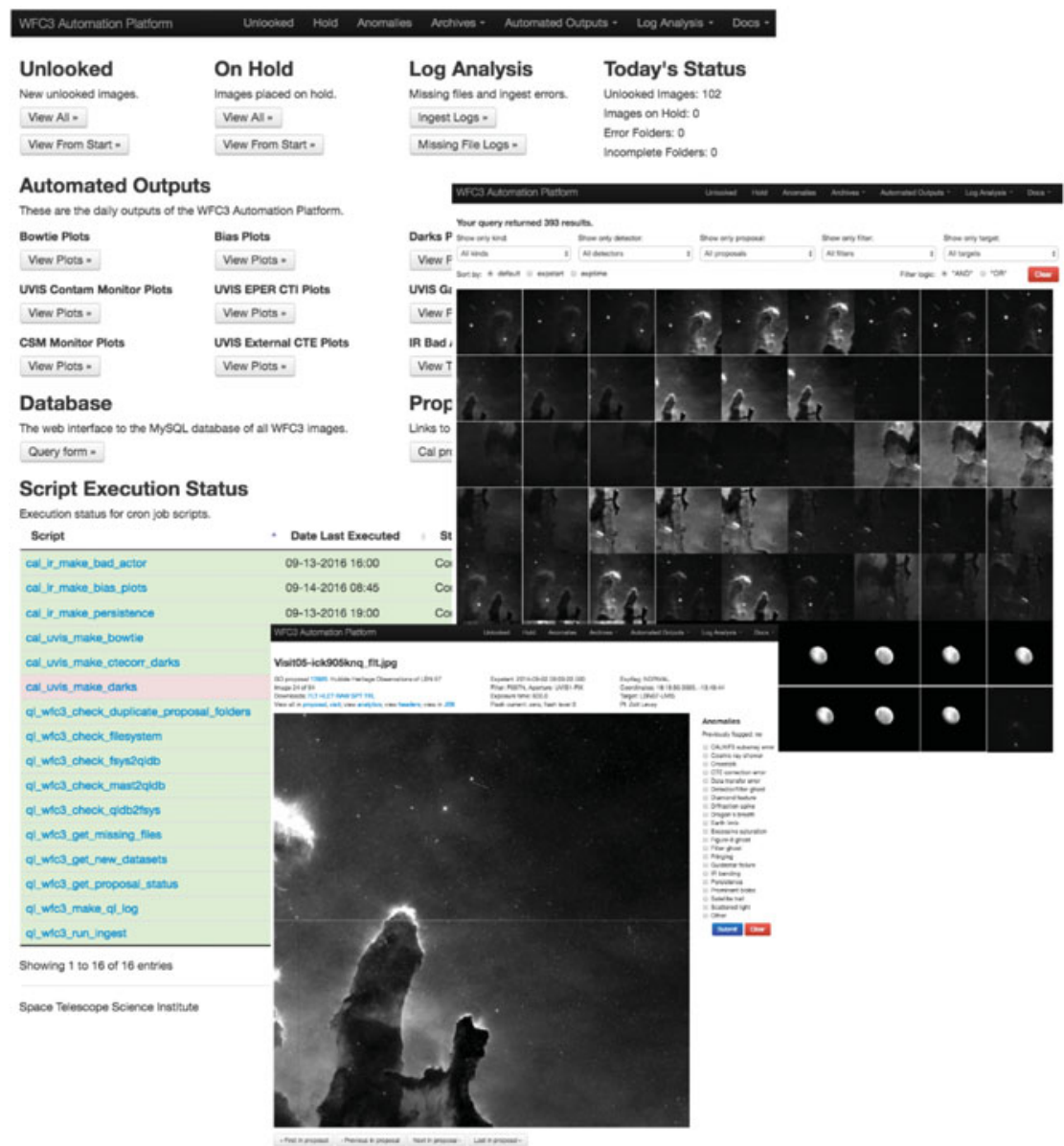

Figure 1. Examples of various pages from the Quicklook website: The Quicklook website home page (top), results from an example query of the Quicklook database (middle), and an example webpage for viewing a single image (bottom).

that implements script logging, data downloading, database connections, and filesystem management to support the Quicklook system, and (5) a website that serves as a user interface to these subsystems and the WFC3 data itself. Figure 1 displays screenshots of some of the capabilities of the Quicklook system via the Quicklook website; users can query the Quicklook database for customized datasets, inspect images, retrieve image metadata, view instrument monitoring plots, flag anomalous images, view the execution status of the automated scripts, and so on.

In this paper, we focus on some of the large-scale WFC3 calibration projects that the Quicklook project has enabled. For further details on how each of the Quicklook subsystems is built and maintained, please see Bourque et al. 2016. Also please note that due to proprietary data, the Quicklook website and system components are only available to WFC3 instrument team members.

\section{Instrument and Quicklook System Monitoring}

With all on-orbit WFC3 calibration data located on centrally-accessible disks as part of the Quicklook filesystem, and an automation platform that executes calibration and monitoring scripts daily, the Quicklook system makes it simple to create and view 

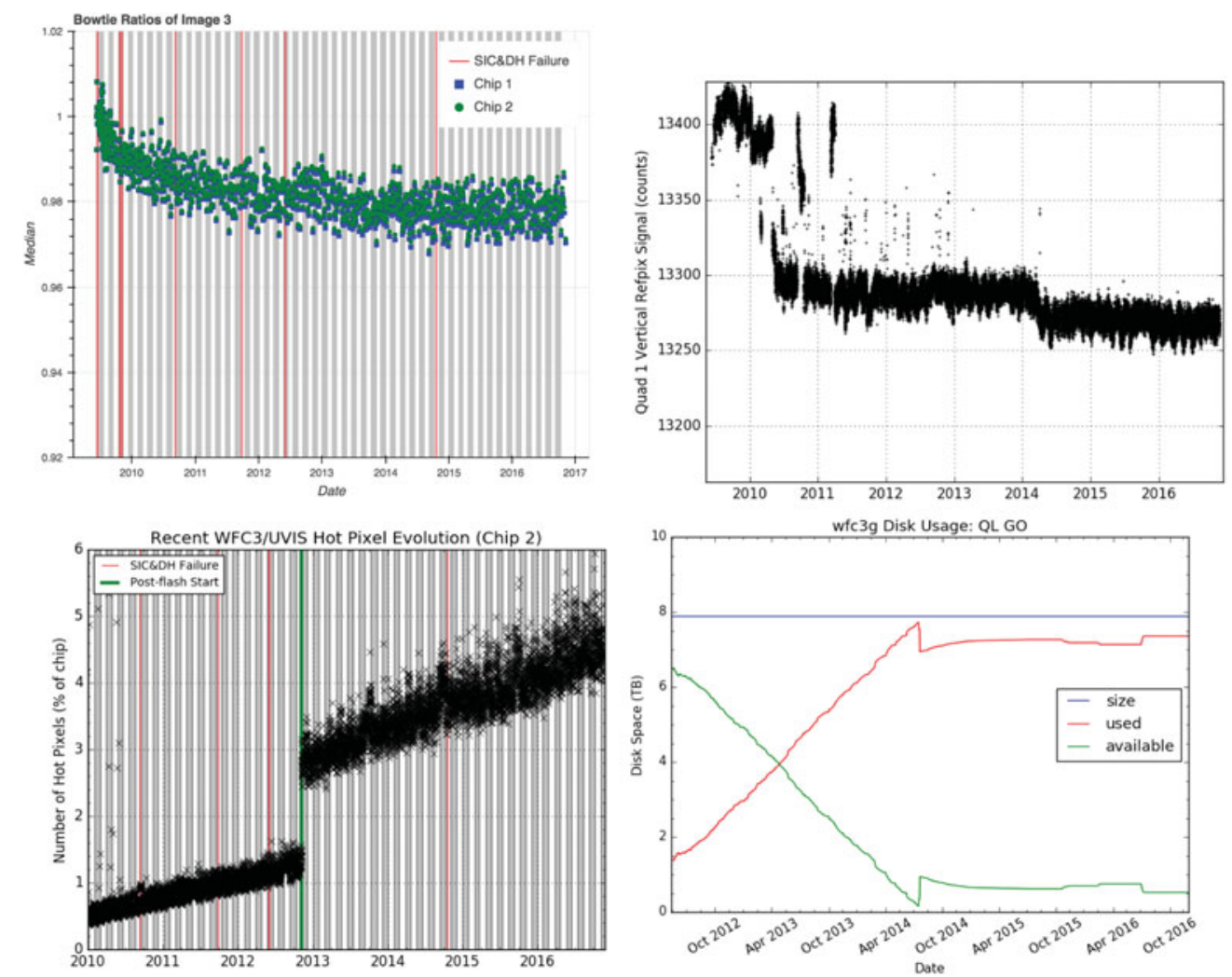

Figure 2. Example instrument monitoring plots that are automatically generated by the Quicklook system on a daily basis: (top left) WFC3/UVIS bowtie monitoring (Bourque \& Baggett 2013), (top right) WFC3/IR bias monitoring (Hilbert \& Petro 2012), (bottom left) WFC3/UVIS hot pixel monitoring, and (bottom right) Quicklook filesystem disk space monitoring.

up-to-date plots that diagnose the instrument's health and stability. Instrument monitoring programs that are employed by Quicklook include but are not limited to (1) the WFC3/UVIS bowtie monitoring program (Bourque \& Baggett 2013), (2) WFC3/IR bias monitoring (Hilbert \& Petro 2012), (3) WFC3/UVIS hot pixel and dark current monitoring (Bourque \& Baggett 2016), and (4) WFC3/UVIS and WFC3/IR gain monitoring (Martlin 2016; Gosmeyer \& Baggett 2015). Figure 2 shows an example of some of the monitor plots that are easily accessible via the Quicklook website.

In addition to instrument monitoring, the Quicklook system also employs several monitors to ensure that the Quicklook system itself is operating nominally. Examples of these monitors include (1) tracking of the available storage space of the Quicklook filesystem (see Figure 2, bottom right), (2) checks to ensure that all files in the Quicklook filesystem have corresponding entries in the Quicklook database and vice versa, (3) automatic downloading of any missing files, and (4) logging the outputs each automated script.

\section{PSF Database}

Another project that has been enabled by Quicklook (the filesystem and database in particular) is the calculation and storage of Point Spread Functions (PSFs) for every measurable star (currently $\sim 20$ million) acquired by the WFC3/UVIS detector. The PSF solutions are extracted from the images in the Quicklook filesystem as 21x21 pixel rasters and stored in a MySQL database along with 1-dimensional and 2-dimensional 
Gaussian models, as well as some image metadata. This resource of PSFs have been used to measure how the UVIS PSF changes with the focus of HST (Anderson et al. 2015). Currently, the WFC3 team is exploring ways to provide the PSFs to the user community.

\section{IR Persistence}

Image persistence is a phenomenon that commonly occurs in mercury cadmium telluride ( $\mathrm{HgCdTe}$ ) detectors such as the WFC3/IR detector (Dressel 2016, Section 5.7.9). Persistence takes the form of ghosts or afterglows from earlier exposures (particularly those of targets that saturate or nearly saturate the detector pixels) and thus can adversely affect science exposures (Dressel 2016, Section 7.9.4). However, WFC3/IR persistence has been well modeled and can be corrected for (STScI 2012). The Quicklook system executes an automated script each day to perform a persistence correction for affected WFC3/IR images. Approximately 100,000 images have been processed to date. The correction products are then delivered to the Mikulski Archive for Space Telescopes (MAST) where users can obtain them. This automated script ensures that persistence correction products are generated and delivered to the archive in a timely manner so that users can apply the correction to their newly acquired data.

\section{Conclusions}

This paper summarizes several of the WFC3 calibration projects that have been enabled by the Quicklook system. With all on-orbit WFC3 images on accessible disks accompanied by the image metadata in a queryable form, a robust Python code base to automatically execute calibration and monitoring code, and a website to serve as a user interface to all of the Quicklook byproducts, a substantial amount of time and effort that otherwise would have been used to calibrate and interact with WFC3 data manually is relieved from the instrument scientists and analysts. As the Quicklook system continues to operate (expected through the end of the WFC3 mission), the number of WFC3 related projects supported by the system is expected to grow.

\section{References}

Anderson, J., Bourque, M., Sahu, K., Sabbi, E., \& Viana, A. 2015, WFC3 Instrument Science Report, WFC3/ISR 2015-08, available at http://www.stsci.edu/hst/wfc3/documents/ ISRs/WFC3-2015-08.pdf

Bourque, M., Bajaj, V., Bowers, A., Dulude, M., Durbin, M., Gosmeyer, C., Gunning, G., Khandrika, H., Martlin, C., Sunnquist, B., \& Viana, A. 2016, ASP-CS, submitted

Bourque, M. \& Baggett, S. 2016, WFC3 Instrument Science Report, WFC3/ISR 2016-08, available at http://www.stsci.edu/hst/wfc3/documents/ISRs/WFC3-2016-08.pdf

Bourque, M. \& Baggett, S. 2013, WFC3 Instrument Science Report, WFC3/ISR 2013-09, available at http://www.stsci.edu/hst/wfc3/documents/ISRs/WFC3-2013-09.pdf

Dressel, L. 2016, Wide Field Camera 3 Instrument Handbook, Version 8.0, Sections 5.7.9 and 7.9.4, available at http://www.stsci.edu/hst/wfc3/documents/handbooks/currentIHB/

Gosmeyer, C. \& Baggett, S. 2015, WFC3 Instrument Science Report, WFC3/ISR 2015-14, available at http://www.stsci.edu/hst/wfc3/documents/ISRs/WFC3-2016-13.pdf

Hilbert, B. \& Petro, L. 2013, WFC3 Instrument Science Report, WFC3/ISR 2012-11, pgs. 24-26, available at http://www.stsci.edu/hst/wfc3/documents/ISRs/WFC3-2012-11.pdf

STScI 2012, "Hubble Space Telescope Image Persistence in the IR detector of WFC3", available at http://www.stsci.edu/hst/wfc3/ins_performance/persistence/

Martlin, C. 2016, WFC3 Instrument Science Report, WFC3/ISR 2016-13, available at http://www.stsci.edu/hst/wfc3/documents/ISRs/WFC3-2015-14.pdf 